\title{
Cooperação, aprendizado e anovação na rede de micro e pequenas empresas de software de Londrina (PR)
}

\author{
Fernanda Yumi Tsujiguchi ${ }^{1}$ \\ Marcia Regina Gabardo da Camara²
}

\begin{abstract}
Resumo: O objetivo do artigo é analisar as ações de cooperação, aprendizado e inovação na rede de micro e pequenas empresas de software de Londrina. Os procedimentos adotados envolvem uma pesquisa de campo e uma pesquisa quantitativa de caráter transversal, cuja coleta de dados envolveu 26 empresários do setor de software em 2007, abordando as ações adotadas e os resultados obtidos nos anos de 2005 a 2007. A análise dos resultados permitiu verificar que as ações de cooperação existentes na rede compreendem relações de parcerias e alianças estratégicas em nível local, em atividades de desenvolvimento e comercialização em software. $\mathrm{O}$ aprendizado perfaz o âmbito interno e externo das empresas tendo como fontes principais de informação a área de $\mathrm{P} \& \mathrm{D}$ e/ou produção e os clientes, respectivamente; e há elevado grau de introdução de inovações incrementais em nível de produção/ desenvolvimento, visto que parte significativa das empresas desenvolve softwares sob encomenda, adaptando produtos e serviços às necessidades dos clientes. O estudo também revela que no caso dos altos índices de introdução de processos tecnológicos novos para a empresa, mas já existente no setor e para o setor de atuação, refletem o esforço tecnológico das empresas para incrementar o processo produtivo e inovativo. O estudo conclui que houve fortalecimento das ações em redes, da cooperação e do aprendizado, fatores que permitiram incrementar a inovação e os resultados financeiros das firmas aglomeradas.
\end{abstract}

Palavras-chave: cooperação; aprendizado; inovação; rede de empresas; Software.

\footnotetext{
1 Mestre em Administração pelo PPA-UEL/UEM. Professora de Empreendedorismo da Unoeste. E-mail fernandayt@gmail.com.

2 Doutora em Economia pela FEA/USP. Professora do Curso de Mestrado em Economia Regional - PPE/ UEL e do curso de graduação em Economia/UEL. E-mail mgabardo@uel.br.
} 


\title{
Cooperation, Learning and Innovation Network in Micro and Small Business Software in Londrina (PR).
}

\begin{abstract}
The aim of the article is to discuss cooperation actions, learning process and innovation in the network of micro and small software's companies of Londrina. The transversal quantitative research was realized with 26 entrepreneurs in 2007, relating to 2005 to 2007 . The main results were: the cooperation actions exists in the network evolving partners relation and strategic alliances in local level, Londrina city, in development and commercialization activities in software; the learning organizations use inside and outside information sources the $R \& D$ and clients particularly; and there is elevated level of introduction of incremental innovation in production/development areas respecting expressive parcel of the software companies develop software's on demand, adjusting products and services to the necessities of the clients. The study also reveals that in the case of high rates of introduction of new technological processes for the company, but existing in the sector and the sector of operations reflect the firms' technological efforts to enhance the production process and innovative. The study concludes that there has been strengthening actions in networks, cooperation and learning improved the innovation and financial performance of clustered firms.
\end{abstract}

Keywords: cooperation; learning; innovation; networks; software.

\section{Introdução}

O advento das Tecnologias da Informação e Comunicação - TIC's contribuíram para a reorganização da estrutura produtiva, revolucionando as bases das relações empresariais. As mudanças radicais nas formas de produção e comercialização baseadas na microeletrônica e nas novas TIC's têm promovido o afastamento do modelo de produção em larga escala rumo à especialização flexível de produção e intensiva em conhecimento (Castells 1999).

Frente a essas transformações econômicas, políticas e tecnológicas, a sobrevivência das empresas mediante a forte concorrência e ambivalências do mercado são fatores determinantes para a formação da rede (Lima Filho et al 2006). Para Olave e Amato (2001) essa configuração em rede se dá por meio da cooperação.

As redes de empresas são formadas com o objetivo de reduzir incertezas e riscos a partir da organização das atividades econômicas através da coordenação e da cooperação (Leon 1998). Para Britto (2002), a análise das variadas formas de cooperação produtiva e tecnológica entre empresas tem propiciado 
uma maior compreensão de como se pode alcançar um melhor desempenho competitivo centrado não na empresa individual, mas principalmente na investigação das relações entre as empresas e as demais instituições.

Um aspecto discutido como alicerce estratégico para a viabilidade econômica das redes é a temática da inovação. A rede promove um ambiente favorável ao compartilhamento de informações, conhecimentos, habilidades e recursos essenciais para os processos de inovação (Balestrin \& Vargas, 2004). O próprio processo inovativo é considerado um sistema integrado em rede (Rotthwell 1995).

A inovação é uma ação conjunta e cooperada de diversos atores internos e externos à organização (Richardson 1972). O processo inovativo não acontece isoladamente; na busca por inovações, as firmas procuram estabelecer relações e interagir com outras organizações, pois podem utilizar informações e conhecimentos que se localizam também fora de seu ambiente. A inovação perfaz processos de aprendizado interativo realizado com a contribuição de variados agentes socio econômicos que possuem diferentes tipos de informações e conhecimentos (Lemos 2001).

O conhecimento se tornou a mola propulsora da economia moderna, e mesmo que a empresa continue sendo o espaço central do processo inovador, a importância de formas coletivas de aprendizado (redes de interação) e cumulatividade existente neste processo tornaram-se fatores críticos de sucesso e de competitividade das empresas. Schumpeter (1982) reforça que as estratégias baseadas na inovação se constituem de fonte de vantagens competitivas para as empresas de alta intensidade tecnológica.

Do mesmo modo, as empresas de software participam de um setor dinâmico e de constantes mutações. Em setores de alta tecnologia - a indústria de software, inclusive - a competição é acirrada (Melo \& Branco 1997). Mesmo com o predomínio de grandes corporações em escala mundial, as redes de empresas são reconhecidas como recurso estratégico de competitividade para que as micro, pequenas e médias empresas conjuguem esforços e possam competir de forma mais equilibrada com as grandes (Lima Filho et al. 2006).

O objetivo do artigo é analisar as redes de microempresas de software de Londrina no que tange às ações de cooperação, parcerias e alianças estratégicas existentes. O estudo está estruturado em quatro partes, excluindo a introdução. As próximas seções abordam: aspectos sobre processos de aprendizado e a inovação segundo a perspectiva evolucionária da economia dentro do contexto da rede de empresas de software de Londrina; metodologia do estudo; análise dos resultados obtidos e considerações finais do trabalho. 


\section{Referencial Teórico}

\subsection{Redes de empresas}

Para Marcon e Moinet (2001) a concepção de rede está ligada à noção de sistema, uma perspectiva dinâmica, que considera uma rede que trabalha networking. Segundo Castells (1999), o termo rede designa um conjunto de pessoas ou organizações interligadas direta e indiretamente. Os atores sociais, enquanto nós da rede, possuem maior intensidade e interação. A participação na rede permite otimizar o uso do espaço e do tempo à medida que essas relações forem sendo intensificadas. Para Britto(2002), o conceito de redes de empresas compreende arranjos interorganizacionais com base em vínculos sistemáticos, na maioria das vezes de caráter cooperativo, entre empresas formalmente independentes, que promovem uma forma particular de coordenação das atividades econômicas. Ring e Van de Ven (1994) compreendem as redes como relacionamentos interorganizacionais cooperativos, sendo resultado de mecanismos socialmente constituídos para a ação coletiva, cujos relacionamentos são constituídos e reestruturados por ações e interpretações simbólicas das partes envolvidas no processo.

O conceito de rede na teoria econômica é empregado sob duas perspectivas: na primeira delas o estudo da rede busca compreender a dinâmica de comportamento de diferentes mercados e o foco de análise está nas estruturas da rede. A análise foca o surgimento de rendimentos crescentes ao longo da trajetória de transformação dos diversos mercados em rede e valoriza a interdependência e interação das unidades produtivas presentes na rede, como por exemplo, as indústrias em rede e redes de empresas. O enfoque da segunda abordagem de redes recai sobre as características estruturais da rede, nos seus processos de estruturação e transformação a partir de estímulos internos e externos. Pressupõe que o ambiente está institucionalmente estruturado em função da densidade de vínculos produtivos e tecnológicos estabelecidos entre empresas e instituições. Segundo Britto (2002), a rede permite obtenção de economias de escala e escopo, podem ser uma fonte de oportunidades lucrativas e de redução de custos e é uma estrutura econômica que estabelece padrões de relações entre os atores.

Para Hoffmann (2005), as redes podem ser classificadas em estruturas formalizadas e informais. As estruturas formalizadas têm como base o escopo contratual em que há o estabelecimento de mecanismos formais para gerir regras de conduta dos agentes. As redes informais têm alicerce não contratual e têm como fundamento a confiança gerada entre os membros, empregando sanções às empresas que violam as normas do grupo. Este último tipo de configuração de rede permite encontros informais entre os atores econômicos que trocam experiências e informações, favorecendo um ambiente de cooperação 
e relações interempresariais mais estruturadas. (Marcon \& Moinet 2001)

A atuação conjunta e cooperada entre empresas de menor porte tem evidenciado a configuração em rede como estratégia para a eficiência produtiva e a competitividade (Hofman 2005). As empresas complementam-se em termos técnicos (produtivos), comerciais (redes de distribuição) e também podem se apoiar no sentido de criar uma central de compras que beneficie o arranjo.

Ebers e Jarillo (1998) reforçam que por intermédio das redes uma empresa está apta a obter e sustentar vantagem competitiva a partir: i) aprendizado mútuo, sustentando desenvolvimento de produtos; ii) co especialização, em que as empresas se tornam lucrativas em novos nichos de mercado; iii) melhor fluidez informacional, favorecendo o fluxo de recursos entre empresas e reduzindo incertezas nas relações; e iv) economias de escala, como reflexo de investimentos conjuntos em P\&D, desenvolvimento de produtos, entre outros.

Para Ribault, Martine e Lebidois (1995), há inúmeras vantagens associadas à configuração de empresas em rede: i) elas podem aprofundar uma especialização, tornando possível a perenidade de todo o know how das atividades; ii) as empresas da rede são um reflexo da atividade econômica da própria rede; e iii) as empresas por escolherem-se por afinidade podem se constituir em uma rede profundamente original em relação às empresas concorrentes, conferindo a si mesmas um elevado grau de exclusividade.

\subsection{Parcerias e alianças estratégicas}

As alianças estratégicas englobam acordos formais e informais entre empresas e permitem o intercâmbio de informações e a aglutinação de competências (Britto, 2002). Geralmente são arranjos cooperativos em estágio pré-competitivo que favorecem aos agentes a possibilidade de exploração de oportunidades tecnológicas e mercadológicas. Com o tempo, tendem a estabelecer relações mais estáveis como forma de aumentar a eficiência operacional.

A aliança é vista como um processo e desta forma está atrelada à necessidade dos agentes das organizações em conhecer as condições de investimentos e o tipo de estrutura de governança existente para se estabelecer um relacionamento. A eqüidade e a eficiência influenciam a continuidade ou não do relacionamento (Ring \& Van de Ven 1994).

Nas alianças estratégicas, as empresas buscam dividir recursos e competências e a redução de custos operacionais (Klotzle 2002). As alianças estratégicas são acordos nos quais os parceiros se comprometem a atingir um objetivo comum pela aglutinação de capacidades e recursos sob certo grau de coordenação 
estratégica e operacional das atividades e pode compreender: atividades de P\&D conjuntas; transferência de tecnologia; concessão de direitos exclusivos para produzir e vender e acordos de cooperação na área de marketing(Teece 1992).

As alianças podem envolver empresas grandes, médias e pequenas que se unem para atuar em mercados selecionados (Ribault; Martinet \& Lebiedois; 1995). Elas podem ser classificadas como: i) alianças oportunísticas ou joint ventures para constituição de um novo negócio, ou ampliar um já existente - as organizações veem uma oportunidade para obterem algum tipo de vantagem competitiva imediata; e ii) alianças de parcerias, que envolvem parceiros (stakeholders - fornecedores e funcionários e consumidores) no processo de negócios (business process) nos diferentes estágios de criação de valor (Kanter 1990). Neste tipo de entrelaçamento há a divisão de recursos e competências e redução de custos operacionais.

Para Faulkner (1992), a aliança estratégica é uma forma de organização das atividades onde se verifica maior grau de integração do que outras formas de cooperação entre os agentes. As alianças estratégicas incrementam as estratégicas competitivas das empresas participantes do arranjo, por meio do intercâmbio mútuo e benéfico de tecnologias, qualificações ou produtos (Yoshino \& Rangan 1997).

O caráter estratégico das ligações entre os pontos da rede em alianças estratégicas, compreendendo cooperação e competição, é o seu principal traço distintivo (Narula \& Hagessorn 1999). O aspecto estratégico deste tipo de arranjo cooperativo associa-se aos objetivos estratégicos das firmas envolvidas que influenciam de forma determinante a conformação da estrutura do arranjo interorganizacional e a relação deste com o ambiente em que está inserido. Para Ring e Van de Ven (1994), a confiança é fator crítico no relacionamento entre parceiros, porque investimentos de longo prazo em negócios e possíveis mudanças que podem ocorrer não são especificados ou controlados anteriormente pelas partes em acordos.

\subsection{Processos de aprendizado}

O aprendizado compreende um processo acumulativo em que organizações adquirem e ampliam o estoque de conhecimentos e buscam o aprimoramento de suas habilidades a fim de desenvolver, produzir e comercializar bens e serviços. (REDESIST 2005)

Malerba (1992) destaca quatro características para analisar o fenômeno do aprendizado tecnológico: i) o aprendizado é um "processo orientado" realizado no interior da firma que envolve um custo específico e orienta a capacitação dos agentes; ii) o aprendizado se constitui por intermédio de fontes internas 
e externas às firmas; iii) é considerado um processo cumulativo de conhecimentos e intertemporal, o que significa que a firma constantemente amplia suas capacitações distintamente de outros agentes; e iv) o aprendizado produz inovações incrementais por meio da eficiência produtiva de processos e possibilita a identificação de oportunidades produtivas e tecnológicas como as inovações radicais e a expansão para novos mercados.

O aprendizado é um processo de constituição de novas competências e obtenção de vantagens competitivas, o qual, pela repetição, experimentação e busca de novas fontes de informação, capacita tecnologicamente as firmas e incrementa as atividades produtivas e inovativas (Campos et al 2002). Torres et al. (2004) afirmam que a forma pela qual cada agente econômico adquire conhecimento é baseada na cooperação que acontece sob distintas formas de interação. Existem os processos de aprendizado em que há: a) o acúmulo de conhecimentos, experiências e práticas acumuladas que circunscreve diretamente as atividades de produção (learning by doing); b) a exploração do uso de determinado produto ou serviço, sendo este e o primeiro processos que ocorrem no interior das firmas e relacionados à eficiência produtiva (learning by using); e c) a interação de outros agentes, como fornecedores e clientes, entre outros, e acontece externamente à firma, o chamado learning by interacting que disponibiliza novas possibilidades tecnológicas e eleva o potencial inovativo (Britto 1999).

Britto (2001) acrescenta que há dois tipos de aprendizado em nível de rede de firmas: informal e formal. O aprendizado do tipo informal ocorre quando há o intercâmbio sistemático de informações e conhecimentos, e a transferência de tecnologias entre os integrantes da rede possibilita diminuir as lacunas do processo inovativo. No aprendizado do tipo formal observa-se a criação intencional de conhecimento tecnológico, compreendendo esforços deliberados de P\&D em que os agentes se unem para criar novas tecnologias. São constituídos de projetos particulares com responsabilidades definidas de cada agente, contemplam uma certa divisão do trabalho quanto a atividades de $\mathrm{P} \& \mathrm{D}$, possibilitando a divisão de resultados e elevando o potencial inovativo.

Quanto aos impactos relativos aos mecanismos informais e formais de aprendizado, os processos informais de aprendizado promovem um equilíbrio nos padrões técnicos produtivos dos agentes ligados à rede, principalmente quanto à capacitação dos recursos humanos; possibilita a troca de informações relativas a processos operacionais e produtivos devido ao intercâmbio de modernas técnicas organizacionais e colabora com a definição de padrões de controle de qualidade e normas técnicas (Britto 2001). Entre os principais impactos dos processos formais de aprendizado destaca-se a redução dos riscos relativos à atividade inovativa. A interação sistemática possibilita a melhor identificação de áreas tecnológicas mais promissoras e, consequentemente, eleva os níveis de produtividade das atividades de $\mathrm{P} \& \mathrm{D}$, ampliando a especialização do arranjo. $\mathrm{O}$ aprendizado, enquanto processo, permite por 
meio da repetição e experimentação o desempenho mais ágil das tarefas em que as firmas conseguem se desenvolver melhor e mais rápido e possibilita o surgimento de novas oportunidades de produção (Teece \& Pisano 1994)

\subsection{Inovação}

Na concepção schumpeteriana, a invenção é vista como uma ideia para ser incorporada a um produto, processo ou um sistema aperfeiçoado, o que não necessariamente leva a inovações técnicas. Uma inovação, no sentido econômico, apenas se caracteriza como a primeira transação comercial (Freeman 1974).

As inovações realizadas por firmas ou indivíduos são novas criações com significado econômico. As inovações abrangem produtos físicos e serviços e processos tecnológicos ou organizacionais; apenas produtos e processos tecnológicos são considerados inovações materiais, enquanto que os serviços e processos organizacionais são tidos como bens intangíveis (Edquist 2001).

As inovações tecnológicas (produtos e processos) emergem de processos complexos cuja emergência, difusão e translação de conhecimentos científicos e tecnológicos se voltam para a criação de novos produtos e processos produtivos (Edquist 1997). A inovação tecnológica de produto e processo significa a utilização do conhecimento sobre novas formas de produzir e comercializar bens e serviços; a inovação organizacional está relacionada à introdução de novos meios de organizar a produção, distribuição e comercialização de bens e serviços (Cassiolato \& Lastres 2004).

A inovação incremental envolve um processo contínuo de inovações que acontece em qualquer atividade industrial ou de serviços e é dependente de pressões de demanda, influências socio culturais, oportunidades e trajetórias tecnológicas. Ela costuma ser iniciada por invenções e sugestões de engenheiros (learning by doing) e outros diretamente comprometidos com o processo produtivo e pelas iniciativas e propostas de usuários (learning by using) (Freeman \& Perez, 1988).

As inovações incrementais traduzem-se em melhorias nos processos e produtos, compreendem a modificação nos inputs usados e modificações na escala; diferenciação de produto no nível horizontal (mudança nas características do produto para conquista de um novo segmento de mercado) e no nível vertical (melhoramento da qualidade por meio da mudança física das propriedades do produto ou incremento da sua confiabilidade, desempenho ou integração) (Malerba 1992).

As inovações radicais compreendem empreendimentos que surgem da pesquisa deliberada e atividades de desenvolvimento que partem de universidades 
e laboratórios governamentais, sendo caracterizados por movimentos descontínuos; novas linhas de produção e modificações na estrutura industrial e pela criação de novos tipos de demanda. Esse tipo de inovação compreende um processo que conta com mecanismos complexos de feedback e de relações interativas entre ciência, tecnologia, aprendizado, produção, política e demanda (learning by interacting) (Edquist 1997). As mudanças de sistemas tecnológicos provocam impactos de longo alcance e abarcam desde inovações incrementais e radicais a inovações organizacionais e gerenciais, atingindo mais do que uma firma e até mesmo influindo nas várias esferas da economia, dando impulso à geração de novos setores (Freeman \& Perez 1988).

A inovação é considerada um processo não linear que pode envolver, inclusive simultaneamente, conhecimentos resultantes da contratação de recursos humanos, da realização de atividades de treinamento, pesquisa e desenvolvimento (P\&D), e demais atividades e experiências acumuladas pela empresa, a partir de sua própria atuação, e da interação com outros agentes e com o ambiente que a cerca (Lastres \& Cassiolato 2004).

\section{Metodologia}

Para a consecução do objetivo desse trabalho foi pesquisada a rede de empresas de software de Londrina. Em setembro de 2005, o Relatório "Identificação, Caracterização, Construção de Tipologias e Apoio na Formulação de Políticas para os Arranjos Produtivos Locais (APL's)" (terceira e quarta etapas), desenvolvido pela Secretaria de Estado de Planejamento e Coordenação Geral (SEPL), Instituto Paranaense de Desenvolvimento Econômico e Social (IPARDES) e pelas Instituições Estaduais de Ensino Superior (IEES), identificou o aglomerado de empresas de software de Londrina como um potencial arranjo produtivo local, tendo como base os objetivos definidos pelo "Programa de Apoio aos Arranjos Produtivos Locais da Rede APL Paraná" e do "Programa de Desenvolvimento da Produção do Governo do Estado do Paraná” (IPARDES 2005). Os resultados obtidos com esse trabalho anterior permitiram diagnosticar a existência de uma rede de empresas.

Para esta pesquisa realizada em 2007 a metodologia de pesquisa adotada foi quali-quantitativa e a amostra por julgamento (Hair 2005), pois a indústria de software é bastante dinâmica, heterogênea e oferece diferentes tipologias. A seleção das 26 empresas foi realizada com base nas seguintes características: i) empresas especializadas em desenvolvimento de softwares e prestação de serviços de consultorias; ii) empresas cadastradas na Prefeitura de Londrina; iii) vinculadas à Plataforma de Tecnologia da Informação - PLATIN e Associação do Desenvolvimento Tecnológico de Londrina e Região - ADETEC e associados Softex (entidades de representação do setor); iv) participação nas atividades do aglomerado de empresas de software de Londrina; v) membros 
de centros de tecnologia como a Incubadora Internacional de Empresas de Base Tecnológica da Universidade Estadual de Londrina - INTUEL e do Centro Tecnológico de Londrina - CTEC; e vi) a aceitação para participar da pesquisa. Foram aplicados 26 questionários junto aos empresários de software de Londrina obtendo-se um total de 34,6\% de micro empresas e $65,4 \%$ de pequeno porte.

O instrumento foi aplicado pelo contato direto (Richardso 1999), nas empresas de software e dirigido aos sócios proprietários das empresas e no caso de ausência destes, para os responsáveis pela área de projetos cooperados. A pesquisa de corte transversal foi realizada em julho e agosto de 2007.

Empregou-se um questionário baseado no modelo da Redesist (2003). Para identificar as ações de cooperação existentes, o questionário constou da aplicação de uma escala ordinal que indicava o tipo de atividade desenvolvida em parceria e a localização do parceiro - local (cidade de Londrina), estado do Paraná, outros estados do país e também do exterior. A escala continha cinco intervalos: alta intensidade, média, baixa ou nenhuma interação.

Para descrever o processo de aprendizado da rede o questionário compreendeu uma escala ordinal que indicasse o grau de importância da fonte de informação, se o mecanismo de aprendizado é formal ou informal, e se a localização da fonte está em nível local da cidade de Londrina, estado do Paraná, outros estados do país e também do exterior. Para identificar as inovações introduzidas foi dada a opção de introdução ou não da inovação pelas empresas.

Uma das principais limitações que restringiu o campo de análise foi quanto à existência de apenas micro e pequenas empresas para serem selecionadas para a amostra. Os cadastros disponíveis, muito dinâmicos e ao mesmo tempo confusos impossibilitaram uma seleção amostral mais aprimorada. $\mathrm{O}$ estudo prévio realizado por Camara et al.(2006) destacou a possibilidade de existência de 127 empresas no cadastro da Prefeitura Municipal de Londrina, representado a amostra $20,5 \%$ do universo.

Uma segunda dificuldade foi a rotatividade de empresas no aglomerado de software de Londrina, fator que impossibilita a generalização dos resultados para o universo total de empresas de software de Londrina ou para outras aglomerações de software.

A terceira limitação foi quanto à definição da atividade econômica principal das empresas, pois algumas delas possuíam mais de um Cadastro Nacional de Pessoa Jurídica - CNPJ- informando códigos distintos, o que impediu uma caracterização mais precisa das empresas em termos de atividade econômica.

As tentativas de correlacionar variáveis do trabalho para realizar testes esta- 
tísticos não obtiveram resultados satisfatórios, visto que não se atingiu níveis de correlação necessários.

Apesar das limitações quanto ao pouco número de empresas para compor a amostra, o que restringiu o campo de análise, e também a rotatividade das empresas na rede de software, a pesquisa colaborou para aprofundar os conhecimentos da indústria de software da cidade de Londrina no que diz respeito às ações de cooperação para elevar a capacidade de produção e de inovação das empresas e seus reflexos sobre a dinâmica competitiva da rede.

\section{Análise e discussão dos resultados}

As empresas da amostra deste artigo foram caracterizadas conforme o tamanho (de acordo com o faturamento anual) e o ramo de atividade. Das 26 empresas pesquisadas, 34,6\% delas são microempresas e 65,4\% são de pequeno porte, conforme Tabela 1.

TABELA 1. TAMANHO DE ACORDO COM O FATURAMENTO ANUAL

\begin{tabular}{l|c|r|c}
\hline $\begin{array}{l}\text { 1. Faixa de Faturamento } \\
\text { Anual }\end{array}$ & $\mathbf{f}$ & \% & Tamanho \\
\hline Até 108.000 & 7 & 26,9 & Micro \\
De 108.001 a $216.00027,7$ & 2 & 7,7 & \\
\hline De 216.001 a 576.000 & 7 & 26,9 & Pequena \\
De 576.001 a 1.200 .000 & 2 & 7,7 & \\
De 1.200 .001 a 1.440 .000 & 1 & 3,8 & \\
De 1.440 .001 a 1.800 .000 & 1 & 3,8 & \\
De 1.800 .001 a 10.500 .000 & 6 & 23,1 & \\
\hline Total & 26 & 100 & \\
\hline
\end{tabular}

FONTE: Pesquisa da autora (julho, 2007)

No que se refere à atividade produtiva das empresas, do total das empresas da amostra, 65\% delas indicaram um percentual de 10 a 50\% dedicados às atividades de desenvolvimento de produtos de software. A Tabela 2 apresenta esses resultados sobre o percentual de desenvolvimento de software nas empresas pesquisadas. 
TABELA 2. RAMO DE ATUAÇÃO DA EMPRESA (PRODUTO)

\begin{tabular}{l|c|c}
\hline Produto de Software (em \%) & f & \% \\
\hline De 10 a $50 \%$ & 17 & 65 \\
De 60 a $100 \%$ & 9 & 35 \\
\hline 1. Total & 26 & 100 \\
\hline
\end{tabular}

FONTE: Pesquisa da autora (2007)

No que se refere ao percentual relativo aos serviços de software realizados pelas empresas, os resultados apontam que a maioria das empresas, $73 \%$ delas, concentram de 46 a $90 \%$ de suas atividades em serviços de software. A Tabela 3 apresenta os percentuais de atuação das empresas em termos de serviços. Verificou-se elevado número de ações de cooperação, do total de empresas pesquisadas: 77\% (20 empresas) afirmaram possuir algum tipo de parceria, aliança estratégica ou contratos de cooperação.

TABELA 3. RAMO DE ATUAÇÃO DA EMPRESA (SERVIÇO)

\begin{tabular}{l|c|c}
\hline Serviço em Software (em \%) & f & \% \\
\hline De 1 a $45 \%$ & 7 & 27 \\
De 46 a $90 \%$ Total & 19 & 73 \\
\hline 2. & 26 & 100 \\
\hline \multicolumn{2}{c}{ FONTE: Pesquisa da autora (2007) }
\end{tabular}

O desenvolvimento de parcerias pela empresa e a localização do parceiro foi significativo. Das empresas que afirmaram a existência de parceria, verificouse ao nível da rede (município de Londrina) que $45 \%$ das empresas indicaram parcerias para comercialização no mercado interno, 40\% em atividades de desenvolvimento, 40\% para obtenção de certificações e 30\% para qualificação de mão de obra. Na esfera estadual, 20\% das empresas apontaram parcerias também em desenvolvimento e $20 \%$ em atividades de comercialização no mercado interno. No Brasil, o dado que mais se destaca é o de comercialização no mercado interno, com 40\% das empresas, e por último, $10 \%$ das empresas declararam possuir parcerias com agentes de origem estrangeira também para assumir uma função do negócio. Verificou-se a predominância de parcerias voltadas à comercialização de produtos/serviços das empresas, conforme os dados da tabela 4 . 
A análise dos resultados revela, em termos de localização de parceiros, a concentração de atividades conjuntas ao nível da rede, ao nível local (comercialização no mercado interno, desenvolvimento e obtenção de certificações), estadual e Brasil (comercialização no mercado interno e desenvolvimento).

\section{TABELA 4. TIPO DE ATIVIDADE X LOCALIZAÇÃO DOS PARCEIROS}

\begin{tabular}{llllllllll}
\hline \multicolumn{1}{c}{ Descrição } & \multicolumn{2}{c}{ Rede } & \multicolumn{2}{c}{ PR } & \multicolumn{3}{c}{ BR } & \multicolumn{2}{c}{ Exterior } \\
& $\mathbf{f}$ & $\mathbf{0}$ & $\mathbf{f}$ & $\mathbf{\%}$ & $\mathbf{f}$ & $\mathbf{\%}$ & $\mathbf{f}$ & $\%$ \\
\hline Outsourcing de Processo de Negócios & - & - & - & - & 2 & 10 & 2 & 10 \\
Outsourcing de TI & 3 & 15 & 2 & 10 & 3 & 15 & - & - \\
Outsourcing Avançado (P\&D) & - & - & 1 & 5 & 2 & 10 & - & - \\
Desenvolvimento & 8 & 40 & 4 & 20 & 4 & 20 & - & - \\
Comercialização no Mercado Interno & 9 & 45 & 4 & 20 & 8 & 40 & - & - \\
Exportação & 1 & 5 & - & - & 1 & 5 & 1 & 5 \\
Qualificação de Mão de Obra & 6 & 30 & 1 & 5 & 1 & 5 & 1 & 5 \\
Obtenção de Certificações & 8 & 40 & - & - & - & - & - & - \\
Outros & 2 & 10 & 1 & 5 & - & - & - & - \\
\hline
\end{tabular}

FONTE: Pesquisa dos autores (2007)

A análise dos resultados revelou que 92\% (24) das empresas pesquisadas participaram nos últimos anos (2005 a 2007) em ações de cooperação formais ou informais com outras empresas ou organizações. Verificou-se o envolvimento das empresas em atividades cooperativas, o que reforça o que Britto (2002) afirma sobre as redes de empresas, que enquanto arranjo organizado estão capacitadas a responder aos estímulos do ambiente, pois estão estruturadas pelas relações tecnológicas e produtivas formais e informais estabelecidas entre empresas e instituições. Conforme Human e Provan (1997 apud Balestrin \& Vargas 2004), há relativa sinergia de ações viabilizadas por interações entre as 20 ou mais empresas de software de Londrina orientadas a atingir objetivos comuns.

Na Tabela 5, verificou-se significativa intensidade da interação das empresas pesquisadas com outras empresas ou organizações, classificando a cooperação (formal, informal ou mista) e a localização do parceiro. Destaca-se que 100\% das empresas apresentaram boa (alta/média/baixa) interação com clientes da cidade de Londrina, estado do Paraná e também com outros estados do país, perpassando relações de caráter formal (contratual) e misto. As relações formais de cooperação entre os clientes demonstram o caráter formal das relações em que há interação não só regular, mas com objetivos e resultados 
esperados da interação. Além de alcançar um benefício mútuo nas interações, os agentes (empresas de software e clientes) definem um método e um objetivo a ser alcançado, o que, provavelmente, de acordo com a pesquisa envolve relações contratuais para a comercialização e desenvolvimento de produtos/serviços.

96\% (alta/média/baixa) das empresas apresentaram forte interação com outras empresas de software de Londrina e também do estado, prevalecendo relações mais informais (2005 a 2007). Nas redes de PME's a coordenação das atividades pode estar sendo realizada pelas próprias empresas, coordenação menos formal, pois é facilitada pela dinâmica de interação dos próprios agentes.

\section{TABELA 5. INTERAÇÃO X FORMALIZAÇÃO X LOCALIZAÇÃO (\%)}

\begin{tabular}{|c|c|c|c|c|c|c|c|c|c|c|c|}
\hline \multirow[b]{2}{*}{ Descrição } & \multicolumn{4}{|c|}{ Intensidade da Interação em \% } & \multicolumn{3}{|c|}{ Formalização } & \multicolumn{4}{|c|}{ Localização } \\
\hline & Alta & Média & Baixa & Sem & Formal & Misto & Informal & $\mathbf{R}$ & PR & BR & $\mathbf{E}$ \\
\hline Fornecedores & 27 & 31 & 34 & 8 & 50 & 4 & 38 & 38 & 30 & 46 & 7 \\
\hline Clientes & 69 & 27 & 4 & - & 46 & 27 & 27 & 77 & 88 & 77 & 7 \\
\hline Concorrentes & 8 & 11 & 31 & 50 & 8 & 4 & 38 & 31 & 19 & 19 & - \\
\hline Outras Empresas de Software & 31 & 31 & 34 & 4 & 27 & 19 & 50 & 69 & 42 & 23 & - \\
\hline Consultores Especializados & 19 & 39 & 27 & 15 & 38 & 8 & 38 & 50 & 35 & 31 & - \\
\hline Universidades & 23 & 23 & 19 & 35 & 54 & 4 & 8 & 62 & 23 & 15 & - \\
\hline Incubadoras de Empresas & 12 & 12 & 15 & 61 & 23 & 8 & 8 & 35 & - & 4 & - \\
\hline Institutos de Pesquisa & 4 & 4 & 15 & 77 & 8 & - & 11 & 12 & 12 & 4 & - \\
\hline Centros de Capacitação & 8 & 19 & 35 & 38 & 46 & - & 15 & 62 & 4 & 7 & - \\
\hline Instituições de Testes/Certific. & 16 & 19 & 19 & 46 & 50 & 4 & - & 19 & 15 & 27 & - \\
\hline Entidades de Representação & 12 & 15 & 15 & 58 & 27 & - & 15 & 11 & - & 30 & - \\
\hline Entidades Sindicais & - & 8 & 34 & 58 & 19 & - & 23 & 38 & - & 4 & - \\
\hline Órgãos de Apoio e Promoção & 8 & 34 & 34 & 24 & 50 & 8 & 19 & 65 & 35 & 12 & - \\
\hline Agentes Financeiros & 19 & 12 & 15 & 54 & 46 & - & - & 12 & 4 & 31 & - \\
\hline
\end{tabular}

FONTE: Pesquisa dos autores (2007)

Constataram-se escassos laços multilaterais com outras instituições; baixa ou nenhuma intensidade de interação com outras organizações, tais como: universidades, institutos de pesquisa (Instituto Agronômico do Paraná (IAPAR), Instituto de Tecnologia do Paraná (TECPAR)), incubadoras de empresas (INTUEL), centros de qualificação profissional, instituições de testes e certificações, entidades de representação (Associação das Empresas Brasileiras de Tecnologia da Informação, Software e Internet (ASSESPRO), Associação para Promoção da Excelência do Software Brasileiro (Softex)) , sindicatos, órgãos de apoio e promoção (SEBRAE, Adetec, Sistema FIEP) e entidades financeiras (FINEP, CEF, BNDES, BRDE), o que demonstra pouca abertura das empresas a estabelecer relacionamentos com outras organizações externas que possibilitem melhor capacitá-las produtiva e tecnologicamente. Algumas empresas indicaram que a cooperação com as universidades locais, em Londrina é restrita: cessão de softwares para alguns departamentos para uso e avaliação do produto (administração, economia e contabilidade); convênios 
para estágio e realização de palestras sobre o setor.

Mesmo que a indústria de software seja enquadrada como de alta tecnologia, existem atividades que são desenvolvidas pelas empresas que são fundamentalmente comoditizadas e de baixo conteúdo de conhecimento e de tecnologia, como no caso das atividades de desenvolvimento e outsourcing . Esta constatação pode explicar a escassa relação das empresas de Londrina com o sistema de Ciência, Tecnologia e Inovação local e nacional.

Na Tabela 6, entre 2005 e 2007, verificou-se que houve 58\% de grau bom (alta/média/baixa) de utilização da cooperação em processos de controle de qualidade e certificação e 69\% em desenvolvimento de produtos e serviços. Apenas 25\% das empresas cooperam para exportar produtos; $31 \%$ das empresas buscam a cooperação com outras empresas e agentes para obter financiamentos e 38\% para reivindicações políticas e apoio financeiro para a rede.

\section{TABELA 6. GRAU DE UTILIZAÇÃO DAS FORMAS DE COOPERAÇÃO (\%)}

\begin{tabular}{ll|l|l|l|l}
\hline Descrição & \multicolumn{3}{l}{ Grau de Utilização em \% } \\
\hline & Alta & Média & Baixa & Não relevante \\
\hline 1. Compra de insumos e equipamentos & 15 & 15 & 4 & 66 \\
2. Venda conjunta de produtos & 19 & 8 & 23 & 50 \\
3. Desenvolvimento de produtos/serviços e processos & 19 & 27 & 23 & 31 \\
4. Capacitação de recursos humanos & 15 & 18 & 15 & 50 \\
5. Obtenção de financiamento & 4 & 19 & 8 & 69 \\
6. Reivindicações políticas, apoio financeiro para a rede & 19 & 8 & 11 & 62 \\
7. Participação conjunta em feiras/eventos e outros & 15 & 23 & 12 & 50 \\
8. Processos de controle de qualidade e certificação & 23 & 23 & 12 & 42 \\
9. Exportação de produtos & - & 13 & 12 & 75 \\
10. Centros de tecnologia como as incubadoras por exemplo & 19 & 12 & 15 & 54 \\
11. Fóruns de discussão & 8 & 23 & 31 & 38 \\
\hline
\end{tabular}

FONTE: Pesquisa dos autores (2007)

A tabela 7 apresenta a avaliação das empresas quanto aos resultados das ações conjuntas implementadas entre 2005 e 2007. As empresas indicaram 100\% de boa importância para a melhoria na qualidade dos produtos; 89\% para a melhoria nos processos produtivos; $85 \%$ nas condições de comercialização e 92\% a novas oportunidades de negócio. Na amostra, 80\% dos entrevistados indicaram como não relevante os resultados das ações

A melhoria da qualidade dos produtos se deve às relações estabelecidas com outras empresas de software de Londrina, em processos de certificação e qualidade e ao relacionamento próximo com os clientes, tendo em vista o perfil das empresas que compõem a rede. Predominam parcerias de outsourcing de processos de desenvolvimento de softwares, cujas atividades são mais comoditizadas e pouco intensivas em conhecimento, pois 425 das empresas relatam a elevada importância da redução de custos nos projetos cooperados. Para 42\% das empresas, o faturamento de projetos cooperados representou 
um percentual de 1 a 20\% sob os rendimentos da empresa. $58 \%$ das empresas afirmaram ter aumentado o faturamento e $42 \%$ apontou que houve aumento do lucro líquido e 50\% delas declaram que houve um aumento no retorno do investimento de projetos cooperados com relação aos dois últimos anos (2005 a 2007).

\section{TABELA 7. AVALIAÇÃO DOS RESULTADOS DAS AÇÕES CONJUNTAS}

\begin{tabular}{|c|c|c|c|c|}
\hline \multirow{2}{*}{ Resultados das Ações Conjuntas } & \multicolumn{4}{|c|}{ Grau de Importância em \% } \\
\hline & Alta & Média & Baixa & Nenhuma \\
\hline $\begin{array}{l}\text { - } \text { Melhoria na qualidade dos produtos } \\
\text { - } \quad \text { Desenvolvimento de novos produtos } \\
\text { - } \text { Melhorias nos processos produtivos } \\
\text { - } \text { Melhor capacitação de recursos humanos } \\
\text { - } \text { Melhorias nas condições de comercialização } \\
\text { - } \quad \text { Introdução de inovações organizacionais } \\
\text { - } \quad \text { Provas oportunidades de negócio } \\
\text { - } \quad \text { Maior inserção da empresa no mercado externo }\end{array}$ & $\begin{array}{l}46 \\
23 \\
46 \\
23 \\
19 \\
27 \\
19 \\
31 \\
31 \\
4\end{array}$ & $\begin{array}{l}42 \\
42 \\
39 \\
27 \\
31 \\
43 \\
31 \\
42 \\
23 \\
8\end{array}$ & $\begin{array}{l}12 \\
12 \\
4 \\
19 \\
31 \\
15 \\
27 \\
19 \\
31 \\
8\end{array}$ & $\begin{array}{l} \\
23 \\
11 \\
31 \\
19 \\
15 \\
23 \\
8 \\
15 \\
80\end{array}$ \\
\hline
\end{tabular}

FONTE: Pesquisa dos autores (2007)

A respeito das inovações introduzidas pelas empresas, observa-se que a introdução de produtos/serviços novos para a empresa, mas já existentes, no mercado, e a introdução de produtos/serviços novos para o mercado nacional revelam o potencial inovativo das empresas de software selecionadas para a amostra, com 69\% em ambos os casos. Quanto ao mercado internacional, apenas $11 \%$ das empresas afirmaram a introdução de produtos/serviços novos nos anos 2005 a 2007. A respeito da introdução de processos tecnológicos novos para a empresa, mas que já existiam no setor, $81 \%$ das empresas apresentaram $81 \%$ esforços inovativos e $77 \%$ apresentaram introdução de processos tecnológicos novos para o setor de atuação, dados que refletem o esforço inovativo das empresas para incrementar o processo produtivo e inovativo nos anos de 2005 a 2007. Sobre implementação de técnicas avançadas de gestão, 35\% das empresas aplicaram alguma forma diferenciada de gestão empresarial. Pode-se compreender esforços de entidades do setor como o Softex, que desenvolve ações de capacitação empresarial no âmbito dos Núcleos Softex distribuídos pelo país (Araújo 2003; Softex 2003).

A análise dos resultados revela que em 39\% da amostra pesquisada há implementação de mudanças significativas nos conceitos e/ou práticas de comercialização com $50 \%$ do total das empresas. O percentual baixo de mudanças na área de marketing e comercialização das empresas denota, segundo Kubota (2005), os fatores críticos de sucesso das empresas de software: bons produtos, liderança empresarial capacitada a administrar uma equipe e ações de Marketing que possibilitem a manutenção das empresas no mercado. As 
empresas de software necessitam gerenciar o relacionamento com os clientes de forma a garantir demandas no longo prazo.

Quanto à implementação de mudanças significativas na estrutura organizacional 58\% indicaram de mudanças na estrutura das empresas, o que reflete a afirmação de Lemos (2001) sobre as especificidades das MPME's: possuem maior capacidade de flexibilidade organizacional e estruturas menos hierárquicas e burocratizadas. Para Lemos (2001), a flexibilidade colabora com a tomada de decisões dos empresários e facilita mudanças no processo produtivo, propiciando condições para a inovação.

A Tabela 8 mostra as inovações realizadas pelas empresas entre 2005 e 2007.

TABELA 8. INOVAÇÕES REALIZADAS PELAS EMPRESAS DE SOFTWARE DE LONDRINA EM PRODUTOS/PROCESSOS E INOVAÇÕES ORGANIZACIONAIS.

\begin{tabular}{ll|l|l}
\hline Descrição & \multicolumn{2}{c}{ Inovações } \\
\hline \multicolumn{2}{l}{ \% } \\
\hline 1. Introdução de produtos/serviços novos para a empresa, mas existente no mercado. & 18 & 69 \\
2. Introdução de produtos/serviços novos para o mercado nacional. & 18 & 69 \\
3. Introdução de produtos/serviços novos para o mercado internacional. & 3 & 11 \\
4. Introdução de processos tecnológicos novos para a empresa, mas já existente no setor. & 21 & 81 \\
5. Introdução de processos tecnológicos novos para o setor de atuação & 20 & 77 \\
6. Implementação de técnicas avançadas de gestão. & 9 & 35 \\
7. Implementação de significativas mudanças na estrutura organizacional & 15 & 58 \\
8. Implementação de mudanças significativas nos conceitos e/ou práticas de marketing & 10 & 39 \\
9. Implementação de mudanças significativas nos conceitos e/ou práticas de comercialização & 13 & 50 \\
10. Implementação de novos métodos e gerenciamento, visando a atender normas de certificação & 12 & 46 \\
& (MPS-BR, CMM, ISO, etc). & \\
\hline
\end{tabular}

FONTE: Pesquisa dos autores (2007)

A implementação de novos métodos e gerenciamento visa a atender normas de certificação segundo $46 \%$ da amostra, 12 empresas - entre elas, cerca de seis empresas da rede participaram de uma ação coletiva para implementar o programa de certificação MPS-BR e duas empresas obtiveram o certificado MPS-BR nível 6.

As micro e pequenas empresas de software selecionadas do município de Londrina recorrem para o incremento da atividade produtiva e inovativa de fontes internas (learning by doing) à empresa, indicando 100\% de boa (alta/ média/baixa) importância ao departamento de P\&D e/ou área de produção. Esse quadro revela, segundo Torres et al. (2004) e Britto (2001, 1999) é que o acúmulo de conhecimentos, práticas e experiências das empresas provem do pessoal da área de produção de software, como engenheiros e técnicos, que funcionam como fonte alimentadora para eficiência produtiva de processos e implementação de mudanças técnicas. 
Os mecanismos informais do aprendizado prevalecem no processo de aprendizado dentro das empresas de software selecionadas, as empresas buscam um equilíbrio nos padrões técnicos produtivos no que se refere à capacitação dos recursos humanos, promovendo a troca de informações de processos operacionais e produtivos e de técnicas organizacionais, e contribuindo com a definição de padrões de controle de qualidade e normas técnicas.

No que se refere a fontes externas de informação e aprendizado, as empresas destacaram com 100\% de alta/média importância os clientes/usuários do produto, concentrando-se com valores equivalentes em nível local, estadual e nacional. Predominam empresas desenvolvedoras de softwares sob encomenda (16 empresas) na rede estudada, que permitem intensa interação produtor/usuário. Por intermédio do learning by using, as empresas interagem com usuários para elevar os níveis de capacitação produtiva, tecnológica e organizacional das empresas. Afirmam ainda que através do feedback dos clientes/usuários é possível identificar desvios, resolver problemas de produtos e processos e explorar oportunidades tecnológicas e até identificar e possibilitar a geração de novos setores.

Adiciona-se à análise que outras fontes externas de aprendizado, como outras empresas de software (locais), consultores especializados (locais e Brasil) e concorrentes, conferências, seminários, cursos, workshops e publicações especializadas, feiras, exibições e livrarias (nível Brasil), tiveram papel relevante nos três últimos anos (2005 a 2007) para elevar a capacitação produtiva e inovativa das empresas. Quanto aos mecanismos de aprendizado, observase que com outras empresas de software e concorrentes prevalecem os mecanismos informais, ou seja, voltados ao equilíbrio de padrões produtivos, enquanto que eventos e consultores especializados predominaram enquanto mecanismos formais de aprendizado.

As micro e pequenas empresas estão empreendendo esforços deliberados de $\mathrm{P} \& \mathrm{D}$, recorrendo a fontes de informação como os clientes, consultores e eventos especializados a fim de criar novas tecnologias. Isto pode revelar a existência de projetos particulares de P\&D nas empresas com certa divisão do trabalho e divisão de resultados. A prática contribui para elevar o potencial inovativo do arranjo, com a melhor identificação de áreas tecnológicas mais promissoras e, consequentemente, elevar os níveis de produtividade das atividades de $\mathrm{P} \& \mathrm{D}$ e ampliar a especialização do arranjo. Por fim, outra fonte de informação que apresentou 100\% boa (alta/média/baixa) importância foi o uso da Internet como meio de informação, abrangendo desde a escala local até internacional.

Os resultados também revelam a baixa ou nenhuma importância dada às universidades (locais e nacionais) e institutos de pesquisa (IAPAR - Instituto Agronômico do Paraná, Embrapa), centros de capacitação profissional (CIEE, Platin na promoção de treinamentos e cursos não voltados à certificação, mas 
que abrangem outras áreas da empresa), instituições de testes e certificações e incubadoras locais (INTUEL) como fontes de informação para o aprendizado. A Tabela 9 apresenta as fontes de informação utilizadas pelas empresas, o grau de formalização dos processos de aprendizado e localização dessas fontes.

TABELA 9. FONTES DE INFORMAÇÃO X FORMALIZAÇÃO X LOCALIZAÇÃO -EMPRESAS DE SOFTWARE - LONDRINA -2005/2007

\begin{tabular}{|c|c|c|c|c|c|c|c|c|c|c|c|}
\hline \multirow[b]{2}{*}{ Descrição } & \multicolumn{4}{|c|}{ Grau de Importância } & \multicolumn{3}{|c|}{ Formalização } & \multicolumn{4}{|c|}{ Localização } \\
\hline & Alta & Média & Baixa & Nulo & Formal & Misto & Informal & $\mathbf{R}$ & $\mathbf{P R}$ & BR & $\mathbf{E}$ \\
\hline $\begin{array}{l}\text { 1. Departamento de P\&D e/ou área de } \\
\text { produção }\end{array}$ & 77 & 15 & 8 & - & 23 & 27 & 40 & & & & \\
\hline 2. Área de vendas, marketing & 38 & 27 & 27 & 8 & 19 & 27 & 46 & & & & \\
\hline \multicolumn{12}{|l|}{ Fontes Externas } \\
\hline 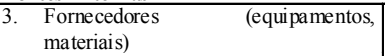 & 23 & 15 & 35 & 27 & 15 & 15 & 42 & 35 & 31 & 42 & - \\
\hline 4. Clientes / Usuários & 77 & 23 & - & - & 31 & 38 & 31 & 81 & 81 & 77 & 7 \\
\hline 5. Outras empresas do setor de software & 35 & 31 & 15 & 19 & 15 & 4 & 61 & 54 & 27 & 42 & 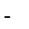 \\
\hline 6. Concorrentes & 19 & 50 & 19 & 12 & 4 & 4 & 81 & 35 & 42 & 73 & 12 \\
\hline 7. Consultores especializados & 38 & 27 & 23 & 12 & 38 & 19 & 31 & 50 & 15 & 50 & - \\
\hline \multicolumn{12}{|l|}{ Universidade e Outras Instituições de Pesquisa } \\
\hline 8. Universidades e Institutos de pesquisa & 19 & 11 & 31 & 39 & 23 & 11 & 27 & 50 & 7 & 15 & - \\
\hline 9. Incubadoras de empresas & 8 & 8 & 27 & 57 & 19 & 11 & 11 & 42 & - & & - \\
\hline $\begin{array}{l}\text { 10. Centros de capacitação profissional, } \\
\text { de assistência técnica e de manutenção }\end{array}$ & 11 & 19 & 39 & 31 & 50 & 4 & 15 & 50 & 23 & 27 & - \\
\hline 11. Instituições de testes e certificações & 27 & 15 & 4 & 54 & 38 & 4 & 4 & 4 & 19 & 23 & - \\
\hline \multicolumn{12}{|l|}{ Outras fontes de informação } \\
\hline $\begin{array}{l}\text { 12. Licenças, registro de } \\
\text { patentes }\end{array}$ & 7 & 4 & 8 & 81 & 15 & 0 & 4 & 4 & 4 & 14 & 4 \\
\hline $\begin{array}{l}\text { 13. Conferências, seminários, cursos, } \\
\text { workshops } \\
\text { especializadas }\end{array}$ & 31 & 34 & 27 & 8 & 46 & 23 & 23 & 46 & 27 & 69 & 7 \\
\hline 14. Feiras, exibições e livrarias & 31 & 27 & 15 & 27 & 31 & 15 & 27 & 27 & 31 & 62 & 7 \\
\hline $\begin{array}{l}\text { 15. Ocasiões sociais (clubes, restaurantes, } \\
\text { bares etc) }\end{array}$ & 23 & 15 & 23 & 39 & 8 & 0 & 54 & 46 & 31 & 31 & - \\
\hline $\begin{array}{l}\text { 16. Assoc. empresariais locais (cons. de } \\
\text { exportação) }\end{array}$ & 7 & 23 & 35 & 35 & 31 & 4 & 31 & 62 & 15 & 7 & - \\
\hline 17. Catálogos, revistas e Sites (Internet) & 54 & 35 & 11 & 0 & 12 & 23 & 65 & 73 & 77 & 92 & 96 \\
\hline
\end{tabular}

FONTE: Pesquisa dos autores (2007)

\section{Considerações Finais}

A pesquisa constatou que as atividades de cooperação existentes na rede correspondem basicamente ao desenvolvimento, comercialização no mercado interno, capacitação de recursos humanos e obtenção de certificação em forma de parcerias e alianças estratégicas com empresas de Londrina e de outras localidades. A interação para cooperação também se dá em maior nível e contatos formais com os clientes/usuários do software para desenvolvimento dos produtos e serviços, e com outras empresas de software da cidade de Londrina, em grau mais informal, para troca de informações e conhecimentos. 
Com relação à identificação do processo de aprendizado coletivo da rede de empresas de software de Londrina, os resultados revelaram que grande parte das empresas recorrem em grau elevado às fontes internas (P\&D e/ou produção) e externas (clientes) majoritariamente para elevar a capacidade produtiva e inovativa das empresas. Como boa parte das empresas focou suas atividades no desenvolvimento do software sob encomenda, acredita-se que as inovações sejam incrementais, pois as fontes de aprendizado perpassam predominantemente a esfera da produção e o contato com usuários do produto.

No caso do pessoal de produção os mecanismos de aprendizado predominantes são mais informais, ou seja, as empresas buscam equilibrar padrões técnicos produtivos quanto à capacitação dos recursos humanos, em que há fluxos e trocas de informações de processos operacionais e produtivos e de técnicas organizacionais contribuindo com a definição de padrões de controle de qualidade e normas técnicas. No caso dos clientes/usuários prevalecem os mecanismos formais de aprendizado. Pode-se dizer que existam esforços mais estruturados por parte das empresas no sentido de deliberar ações que promovam não só o melhoramento de produtos, processos técnicos e organizacionais como também buscar a identificação de novas oportunidades de negócios para beneficiar seus próprios clientes.

O estudo demonstrou a importância num plano secundário de outras fontes externas de informação com mecanismos informais de aprendizado na relação com outras empresas de software (locais) e concorrentes e processos de aprendizado formais com consultores especializados (locais e Brasil) e participação em conferências, seminários, cursos, workshops, publicações especializadas e feiras e exibições (nível Brasil) para melhorar a capacitação produtiva e inovativa das empresas. Isto demonstrou que as empresas estavam buscando de forma sistemática empreender ações intencionais de P\&D para criar novas tecnologias.

Entre 2005 a 2007, as empresas estão mais direcionadas a lançar produtos/ serviços novos para a empresa e também para o mercado nacional. Elas introduziram mais inovações no mercado nacional do que no mercado internacional. O estudo revela que os altos índices de introdução de processos tecnológicos novos para a empresa, mas já existente no setor e para o setor de atuação, refletem o esforço tecnológico das empresas para incrementar o processo produtivo e inovativo. Há a compra de máquinas e equipamentos que implicam em significativas melhorias tecnológicas de produtos/processos ou que estão associados aos novos produtos/processos no período analisado.

Os resultados da pesquisa ratificam ainda as dificuldades em termos de gestão empresarial encontradas pelos empresários do setor, como a pouca implementação de técnicas avançadas de gestão e de mudanças significativas nos conceitos e/ou práticas de marketing e de comercialização estando aliado aos estudos dos fatores que inibem o crescimento da indústria de software 
brasileira, Verificou-se, por outro lado, que a flexibilidade organizacional e a ausência de burocracias excessivas presentes nas MPME's favorecem a implementação de mudanças na estrutura organizacional, facilitando a tomada de decisões e alterações no processo produtivo, o que beneficia as atividades inovativas.

As autoras agradecem ao Fundo Paraná e SETI- Governo do Estado do Paraná pelos recursos financeiros recebidos.

\section{Referências}

ARAÚJO, E.R. (2003). "Oportunidades e desafios para o desenvolvimento de uma indústria de software nacional”. Revista Ciência e Cultura, São Paulo, 55( 2).

BALESTRIN, A.; VARGAS, L.M. A (2004). “Dimensão Estratégica das Redes Horizontais de PME's: Teorizações e Evidências”. Revista de Administração Contemporânea. Edição Especial, 203-227.

BOTELHO, M.R. (1999). "Políticas de Apoio às Pequenas Empresas Industriais no Paraná: Uma avaliação a partir da experiência internacional”. Campina: Tese de Doutorado. Instituto de Economia. Universidade de Campinas.

BRITTO, J. (1999). “Características Estruturais e Modus Operandi das Redes de Fïmas em Condições de Diversidade Tecnológica”. Rio de Janeiro: Tese de Doutorado - Instituto de Economia, Universidade Federal do Rio de Janeiro.

BRITTO, J. (2002). Cooperação interindustrial e redes de empresas. In: KUPFER, D.; HASENCLEVER, L. (orgs). Economia Industrial - Fundamentos Teóricos e Práticas no Brasil. Rio de Janeiro: Elsevier Editora, 345-386.

BRITTO, J. (2001). Cooperação Tecnológica e Aprendizado Coletivo em Redes de Firmas: Sistematização de Conceitos e Evidências Empíricas. Anais do XXIX Encontro Nacional de Economia.

CAMPOS, R.R. \& CARIO, S.A.F. \& NICOLAU, J.A. \& VARGAS, G. (2002). Aprendizagem por interação: pequenas empresas em sistemas produtivos e inovativos locais. Rio de Janeiro. Versão preliminar, disciplina economia da inovação.

CASSIOLATO, José E. \& LASTRES, Helena M. M.(2004). “O foco em arranjos produtivos e inovativos locais de micro e pequenas empresas”. In: LASTRES, H. M. M.; CASSIOLATO, José E. (coord.). Arranjos produtivos locais: uma nova estratégia de ação para o Sebrae. Rio de Janeiro: RedeSist.

CAMARA, M.R.G. \& CAMPOS, M.F.S.S \& SEREIA, V.J.S.(2006) "Políticas públicas para a inovação e a produção na aglomeração de software em Londrina”. In XIII SIMPEP - Bauru.

CASTELlS, M. (1999). A Era da Informação: Economia, Sociedade e Cultura. $3^{\mathrm{a}}$.Ed . vol.1. São Paulo: Paz e Terra .

DOSI, G. (2005). Mudança Técnica e Transformação Industrial. Campinas: Editora 
da UNICAMP.

EBERS, M. \& JARILLO, J.C. (1998)” The construction, forms, and consequences of industry networks". International Studies of Management and Organizational, 27, 3-21.

EDQUIST, C. (1997). Systems of Innovation - Technologies, Institutions and Organizations. New York: Pinter.

FAULKNER, D. (1992). "Strategic alliances: cooperation for competition". In FALKNER, D. \& JOHNSON, G. (eds) The challenge of strategic management. London: Kogan Page.

FREEMAN, C. (1974). “The Economics of Industrial Innovation”. London: Frances Pinter.

FREEMAN, C. (1988). "Introduction”. In DOSI, G. et al. Technical change and economic theory. London: Pinter Publishers, p. 1-12.

FREEMAN, C. \& PEREZ, C. (1988) "Structural crises and adjustment, business cycles and investment behaviour”. In DOSI, G. et al. Technical change and economic theory. London: Pinter, pp.38-66.

GUTIERREZ, R.M.V. \& ALEXANDRE, P.V.M. (2004) Complexo Eletrônico: Introdução ao Software. BNDES Setorial.

HAIR, J.F. \& BABIN, B.\& MONEY, A.H.\& SAMOUEL, P. (2005) Fundamentos de Métodos de Pesquisa em Administração. Porto Alegre: Bookman, 2005.

HOFFMANN, V. E. (2005). Competitividade em Redes de PME's. Texto para discussão. Programa de Mestrado em Administração da Universidade do Vale do Itajaí - RS.

KANTER, R.M. "When giants learn cooperative strategies". (1990). Planning Review $18(1)$.

KUBOTA, L.C. (2005). Desafios para a indústria de software. Brasília: Instituto de Pesquisa econômica Aplicada - IPEA, Ministério do Planejamento, Orçamento e Gestão.

LASTRES, H.M.M.; CASSIOLATO, J.E. (2004). Arranjos Produtivos Locais: Uma estratégia de ação para o Sebrae: Glossário de Arranjos e Sistemas Produtivos e Inovativos Locais. Rio de Janeiro: Universidade Federal do Rio de Janeiro.

LEMOS, C. Inovação em Arranjos e Sistemas de MPME. (2001). Nota Técnica 1.3. Rede de Sistemas Produtivos e Inovativos Locais - RedeSist, Universidade Federal do Rio de Janeiro, Rio de Janeiro.

LEON, M.E. (1998). Uma Análise de Redes de Cooperação das Pequenas e Médias Empresas do Setor das Telecomunicações. Dissertação de Mestrado, Engenharia da Produção da Escola Politécnica da Universidade de São Paulo.

LIMA FILHO, D.O.L. \& MAIA, F.S. \& SPROESSER, R.L., MORAES, F. \& MORAES, R. (2006) Redes de Cooperação no Varejo Alimentar de Vizinhança: Percepções dos Associados. In: Gestão e Produção, (2): 311-24.

MALERBA, F. (1992). "Learning by firms and incremental technical change”. Economic Journal, (102):845-859.

MARCON, C.\& MOINET, N. (2001) Estratégia - Rede. Caxias do Sul: EDUCS - 
Editora da Universidade de Caixas do Sul.

MELO, P.R.S. \& BRANCO, C.E.C. (1997) “Setor de Software: Diagnóstico e Proposta de Ação para o BNDES”. Brasília: BNDES SETORIAL (5): 111 a 127.

NARULA, R. HAGEDOORN, J. (1999). "Innovating through strategic alliances: moving towards international partnerships and contractual agreements", Technovation, (19): 283-294.

OLAVE, M.E.L \& AMATO, J.A. (2001). "Redes de Cooperação Produtiva: Uma estratégia de competitividade para pequenas e médias empresas”. In Gestão e Produção: 2001, (3): 289-303.

REDESIST - REDE DE PESQUISAS EM SISTEMAS PRODUTIVOS E INOVATIVOS LOCAIS - (2005). "Mobilizando Conhecimentos para Desenvolver Arranjos Produtivos e Inovativos Locais de Micro e Pequenas Empresas no Brasil”. Projeto Apoiado pelo Sebrae. Oitava Revisão. URL: www.redesist.ie.ufrj.br/glossario. php. Acesso em: Dezembro de 2005.

REDESIST - REDE DE PESQUISA EM SISTEMAS PRODUTIVOS E INOVATIVOS LOCAIS. (2003). “Questionário para arranjos produtivos locais”. In LASTRES, Helena M. M.; CASSIOLATO, Jorge E. (coord.) Arranjos produtivos locais: uma nova estratégia de ação para o Sebrae. Rio de Janeiro: RedeSist.

RIBAULT, M. \& MARTINET, B. \& LEBIDOIS, D. (1995) A gestão das tecnologias. Coleção gestão \& inovação. Lisboa: Publicações Dom Quixote.

RICHARDSON, G. B. (1972). “The organizational of industry”. Economic Journal, p.883-896.

RICHARDSON, R.J. (1999). Pesquisa Social: métodos e técnicas. São Paulo: Atlas.

RING, P. S. \& VAN de VEN, A. H. (1994). Developmental process of cooperative interorganizational relationships. Academy of Management Review, 19(1): 90-118.

SCHUMPETER, J.A. (1982). A teoria do desenvolvimento econômico. São Paulo: Abril Cultural. (Os economistas).

SOFTEX. Projeto MTI. Softex - W-Class. (2003). A Indústria de Software no Brasil - 2002 - Fortalecendo a Economia do Conhecimento. Campinas: Softex.

TEECE, D.J. (1992). “Competition, cooperation and innovation: Organizational arrangements for regimes of rapid technological progress”. Journal of Economic Behavior and Organization, Amsterdam, 18(1): 1-25.

TEECE, D.J. \& PISANO, G. (1994). The Dynamic Capabilities of Firms: an Introduction. Industrial and Corporate Change, v. 1(3).

TORRES, R. \& ALMEIDA, S. \& TATSCH, A.L. (2004). Nota técnica o8. Projeto de Pesquisa: Aprendizado, Capacitação e Cooperação em Arranjos Produtivos e Inovativos Locais de MPEs: implicações para políticas. Cooperação e Aprendizado em Arranjos Produtivos Locais: aspectos conceituais e indicadores da Redesist. Coord. José E. CASSIOLATO \& Helena M.M. LASTRES. UFRJ, OEA, Redesist.

YOSHINO, M.; RANGAN, U. S. (1996). Alianças estratégicas: uma abordagem empresarial à globalização. São Paulo: Makron Books. 
TSUJIGUCHI, F. Y. \& CAMARA, M. R. G. Cooperação, aprendizado e inovação na rede de micro e ...

Recebido em: 27 de julho de 2010

Primeira resposta em: o6 de janeiro de 2011

Aceite em: 06 de janeiro de 2011 